\title{
Change of Exhaled Acetone Concentration Levels in Patients with Acute Decompensated Heart Failure A Preliminary Study
}

\author{
Tetsuro Yokokawa, ${ }^{1}$ MD, Takamasa Sato, ${ }^{1}$ MD, Satoshi Suzuki, ${ }^{1}$ MD, Masayoshi Oikawa, ${ }^{1}$ MD, \\ Akiomi Yoshihisa, ${ }^{1}$ MD, Atsushi Kobayashi, ${ }^{1}$ MD, Takayoshi Yamaki, ${ }^{1}$ MD, Hiroyuki Kunii, ${ }^{1}$ MD, \\ Kazuhiko Nakazato, ${ }^{1}$ MD, Hitoshi Suzuki, ${ }^{1}$ MD, Shu-ichi Saitoh, ${ }^{1}$ MD, Takafumi Ishida, ${ }^{1}$ MD, \\ Akito Shimouchi, ${ }^{2}$ MD and Yasuchika Takeishi, ${ }^{1}$ MD
}

\begin{abstract}
Summary
Exhaled acetone concentration is one of the expected compounds to be a breath biomarker in heart failure. However, it has not been clarified how exhaled acetone concentration changes in clinical course of heart failure.

To investigate whether exhaled acetone concentration changes after treatment in acute decompensated heart failure (ADHF).

This study included 19 patients with ADHF (ADHF group) and eight patients with stable heart failure (control group). Exhaled acetone was collected from these patients, and the concentration was measured with gas chromatography.

The ADHF group had higher heart rates $(P=0.046)$, higher New York Heart Association class $(P<$ $0.001)$, higher levels of brain natriuretic peptide $(P=0.026)$, blood total ketone bodies $(P=0.015)$, and exhaled acetone concentration $(P<0.001)$, compared with the control group. In ADHF group, exhaled acetone concentration significantly decreased after treatment (median: 2.40 versus $0.92 \mathrm{ppm}, P<0.001)$. However, in the control group, exhaled acetone concentration did not significantly change (median: 0.73 versus $0.49 \mathrm{ppm}, P=$ $0.141)$

In these preliminary findings, exhaled acetone concentration in patients with ADHF drastically decreased by treatment. Serial exhaled acetone measurement might be useful to evaluate the course of ADHF.
\end{abstract}

(Int Heart J 2018; 59: 808-812)

Key words: Ketone body, Breath analysis, Biomarker

$\mathrm{M}$ any low molecular compounds are known in human breath. Some of them are correlated with habits and illness and clinically used in daily practice. For example, breath carbon monoxide is useful marker to assess a smoking status. ${ }^{1)}$ Urea breath test is a simple test in the management of Helicobacter pylori infection. ${ }^{2}$

In cardiovascular diseases, breath analysis has not been used in daily clinical practice. Exhaled acetone concentration is one of the expected compounds to be a breath biomarker in patients with heart failure. ${ }^{3-6)}$ Exhaled acetone concentration is known to be correlated with New York Heart Association (NYHA) class and brain natriuretic peptide in patients with heart failure. ${ }^{3)}$ Moreover, exhaled acetone concentration could have prognostic impact in patients with heart failure." Only two anecdotal reports showed the change of exhaled acetone concentration after treatment in a patient with acute decompensated heart fail- ure (ADHF). ${ }^{8.9)}$ Except anecdotal reports, it has been unknown how exhaled acetone concentration would change in the clinical course of patients with heart failure.

In this study, we investigated whether exhaled acetone concentration would change after treatment in patients with ADHF.

\section{Methods}

This preliminary study prospectively included 19 patients with ADHF who hospitalized between September 2016 and March 2017 in Fukushima Medical University Hospital (ADHF group). Diagnosis of heart failure was evaluated by over two independent cardiologists with two major criteria or one major criterion in conjunction with two minor Framingham criteria. ${ }^{10)}$ Exclusion criteria were patients with hyperglycemic crisis including diabetic ketoacidosis. Eight patients with compensated stable heart

From the ${ }^{1}$ Department of Cardiovascular Medicine, Fukushima Medical University, Fukushima, Japan and ${ }^{2}$ Department of Lifelong Sports for Health Biochemical Sciences, College of Life and Health Sciences, Chubu University, Aichi, Japan.

This work was supported, in part, by a grant from the Japanese Society for the Promotion of Science (No. 15K19401 to T.Y.)

Address for correspondence: Tetsuro Yokokawa, MD, Department of Cardiovascular Medicine, Fukushima Medical University, 1 Hikarigaoka, Fukushima, Fukushima, 960-1295, Japan. E-mail: yokotetu@fmu.ac.jp

Received for publication August 20, 2017. Revised and accepted October 29, 2017.

Released in advance online on J-STAGE May 23, 2018.

doi: $10.1536 /$ ihj. $17-482$

All rights reserved by the International Heart Journal Association. 
failure who hospitalized in Fukushima Medical University Hospital were also enrolled (control group). The control patients were not ADHF on admission. The control patients included three cases of dilated cardiomyopathy, three cases of ischemic cardiomyopathy, one case of sick sinus syndrome, and one case of myocarditis. Baseline data of sex, age, body mass index, NYHA class, etiology of heart failure, medical history, physical findings, and current medications were corrected at the time of first enrollment in this study. NYHA class was also evaluated at a stable phase after heart failure treatment. This study protocol was approved by the institutional ethics committee of the Fukushima Medical University. Written informed consent was provided by all patients.

Blood test and breath analysis: All patients were given the hospital diet with an average total energy count of $1,400-1,800 \mathrm{kcal} / \mathrm{day}$. Breath analysis was performed in the early morning after at least a 12-hour fast. In the ADHF group, breath analysis was performed twice at the day of the first morning after admission (acute phase) and at a stable phase after heart failure treatment. The improvement of heart failure was confirmed by NYHA class, findings of physical examination, chest X-ray and echocardiography, and levels of brain natriuretic peptide. In the control group, breath analysis was performed twice at a stable phase on different days. There was no worsening case of heart failure in all study subjects. Patients blow only once through a breath-sampling bag (Collection Bag, Laboratory for Expiration Biochemistry, Nourishment Metabolism Co., Ltd., Nara, Japan). About $200 \mathrm{~mL}$ of the breath was collected and maintained in the breathsampling bag. The collected breath was transferred to a gas-tight glass syringe, and $5 \mathrm{~mL}$ of the total was injected into a gas analysis device (GC-8A; Shimadzu Co., Ltd., Kyoto, Japan) to measure breath acetone concentration within the day that collected the breath. Every measurement of the breath acetone was calibrated by standard gas (Sumitomo Seika Chemicals Co., Ltd., Osaka, Japan). Acetone concentration was calculated by subtracting the acetone concentration of ambient air around the patients using an identical sampling bag for the breath collection.

Blood sampling was analyzed for estimated glomerular filtration rate, total bilirubin, glucose, hemoglobin A1c, brain natriuretic peptide, and total ketone bodies.

Echocardiography: Echocardiography was performed by a blinded, experienced echocardiographer using the standard techniques. ${ }^{11)}$ The echocardiographic parameters investigated included left ventricular diastolic diameter, left ventricular systolic diameter, and left atrial diameter from the parasternal long-axis view. Left ventricular ejection fraction was measured by modified biplane Simpson's method.

Statistical analysis: Data were analyzed using the Statistical Package for Social Sciences version 24 (SPSS Inc., Chicago, IL, USA). Quantitative data are expressed as mean $\pm \mathrm{SD}$ and median and interquartile range. The statistical significance of differences was analyzed using Students' $t$-test for parametric continuous variables and the Mann-Whitney $U$-test for nonparametric continuous variables. Categorical variables were compared using chisquare test or Fisher's exact test. Correlations were ana- lyzed using Spearman's correlation analysis for variables. The paired samples were analyzed using Wilcoxon singed rank test. $P<0.05$ was considered statistically significant.

\section{Results}

Baseline characteristics: The ADHF group had higher heart rates $(P=0.046)$, higher NYHA class $(P<0.001)$, higher levels of brain natriuretic peptide $(P=0.026)$, blood total ketone bodies $(P=0.015)$, and exhaled acetone concentration at the first time $(P<0.001)$, compared with the control group (Table I). The levels of estimated glomerular filtration rate, total bilirubin, glucose, and hemoglobin A1c and the usage of antidiabetic medications did not have significant difference between groups.

Correlation between exhaled acetone concentration and clinical variables: Exhaled acetone concentration at the first time was significantly correlated with blood ketone bodies $(r=0.767, P<0.001$, Figure 1$)$, NYHA class $(r=0.668, P<0.001)$, and levels of brain natriuretic peptide $(r=0.400, P=0.039)$ in all study subjects. However, exhaled acetone concentration at the first time was correlated with neither body weight $(r=0.210, P=0.294)$, left ventricular ejection fraction $(r=0.121, P=0.548)$, creatinine $(r=0.064, P=0.751)$, nor hemoglobin A1c $(r=$ $0.239, P=0.229)$.

Change of exhaled acetone concentration: The mean intervals between the first and second measurements were 9 days in the ADHF group and 4 days in the control group. In the ADHF group, heart failure was improved after treatment determined by NYHA class, findings of physical examination, chest X-ray and echocardiography, and levels of brain natriuretic peptide. In the ADHF group, NYHA class $(P<0.001)$ and levels of brain natriuretic peptide $(P$ $<0.001)$ were significantly decreased after treatment. Furthermore, exhaled acetone concentration was also significantly decreased after treatment (median: 2.40 versus 0.92 ppm, $P<0.001)$. In contrast, in the control group, NYHA class $(P=1.000)$, levels of brain natriuretic peptide $(P=$ 0.068 ), and exhaled acetone concentration (median: 0.73 versus $0.49 \mathrm{ppm}, P=0.141$ ) did not significantly change (Table II).

The change of exhaled acetone concentration was correlated with the change of blood total ketone bodies ( $r$ $=0.621, P=0.003)$ and the change of NYHA class $(r=$ $0.403, P=0.037)$ but not with the change of brain natriuretic peptide $(r=0.312, P=0.147)$.

Comparison of the amount of change: The change in exhaled acetone concentration from the first to second measurements was significantly greater in the ADHF group, compared with the control group $(P<0.001$; Figure 2).

\section{Discussion}

This preliminary study is the first to show the changes of exhaled acetone concentration after treatment of patients with ADHF. Patients with ADHF had elevated levels of exhaled acetone concentration. As the improvement of heart failure, levels of exhaled acetone concentration significantly decreased in this study. 
Table I. Baseline Characteristics

\begin{tabular}{|c|c|c|c|}
\hline & $\operatorname{ADHF}(n=19)$ & Control $(n=8)$ & $P$ value \\
\hline Age, years & $77 \pm 24$ & $71 \pm 8$ & 0.395 \\
\hline Male & $8(42)$ & $2(25)$ & 0.666 \\
\hline $\mathrm{BMI}, \mathrm{kg} / \mathrm{m}^{2}$ & $19 \pm 2$ & $18 \pm 3$ & 0.243 \\
\hline Smoking history & $10(53)$ & $5(63)$ & 0.696 \\
\hline NYHA class, I/II/III/IV & $0 / 0 / 14 / 5$ & $3 / 4 / 1 / 0$ & $<0.001$ \\
\hline $\mathrm{SBP}, \mathrm{mmHg}$ & $122 \pm 25$ & $120 \pm 16$ & 0.852 \\
\hline Heart rate, beats/minute & $86 \pm 17$ & $68 \pm 17$ & 0.046 \\
\hline \multicolumn{4}{|l|}{ Etiology } \\
\hline Ischemic & $3(16)$ & $3(38)$ & 0.319 \\
\hline Valvular & $3(16)$ & $0(0)$ & 0.532 \\
\hline Cardiomyopathy & 7 (37) & $3(38)$ & 1.000 \\
\hline Others & $6(32)$ & $2(25)$ & 0.558 \\
\hline Hypertension & $14(74)$ & $4(50)$ & 0.375 \\
\hline Dyslipidemia & $12(63)$ & $5(63)$ & 1.000 \\
\hline Diabetes mellitus & $13(68)$ & $4(50)$ & 0.415 \\
\hline \multicolumn{4}{|l|}{ Laboratory data } \\
\hline eGFR, $\mathrm{mL} /$ minute $/ 1.73 \mathrm{~m}^{2}$ & $43 \pm 18$ & $48 \pm 14$ & 0.651 \\
\hline Total bilirubin, mg/dL & $1.0 \pm 0.5$ & $0.8 \pm 0.3$ & 0.309 \\
\hline Glucose, $\mathrm{mg} / \mathrm{dL}$ & $112 \pm 33$ & $108 \pm 10$ & 0.852 \\
\hline Hemoglobin A1c, \% & $6.5 \pm 0.8$ & $6.2 \pm 0.5$ & 0.409 \\
\hline BNP, pg/mL (median, IQR) & $541(292-977)$ & $231(117-542)$ & 0.026 \\
\hline $\mathrm{TKB}, \mu \mathrm{ml} / \mathrm{L}$ (median, IQR) & $326(147-848)$ & $111(34-194)$ & 0.015 \\
\hline \multicolumn{4}{|l|}{ Echocardiography } \\
\hline LVDd, mm & $53 \pm 10$ & $55 \pm 9$ & 0.832 \\
\hline LVDs, mm & $42 \pm 12$ & $43 \pm 11$ & 0.958 \\
\hline LVEF, \% & $42 \pm 18$ & $39 \pm 15$ & 0.710 \\
\hline $\mathrm{LAD}, \mathrm{mm}$ & $46 \pm 9$ & $46 \pm 9$ & 0.915 \\
\hline \multicolumn{4}{|l|}{ Medication } \\
\hline ACE-I/ARB & $9(47)$ & $7(88)$ & 0.090 \\
\hline$\beta$-Blocker & $14(74)$ & $5(63)$ & 0.658 \\
\hline Loop diuretic & $15(79)$ & $5(63)$ & 0.633 \\
\hline Insulin & $4(21)$ & $1(13)$ & 1.000 \\
\hline Oral antidiabetic agents except SGLT-2 inhibitor & $6(32)$ & $2(25)$ & 1.000 \\
\hline SGLT-2 inhibitor & $1(5)$ & $0(0)$ & 1.000 \\
\hline EAC at the first time, ppm (median, IQR) & $2.40(1.62-6.08)$ & $0.73(0.60-0.93)$ & $<0.001$ \\
\hline
\end{tabular}

Values are mean $\pm \mathrm{SD}$, median (IQR), or $n(\%)$. ADHF indicates acute decompensated heart failure; BMI, body mass index; NYHA, New York Heart Association; SBP, systolic blood pressure; eGFR, estimated glomerular filtration rate; BNP, brain natriuretic peptide; IQR, interquartile range; TKB, total ketone body; LVDd, left ventricular diastolic diameter; LVDs, left ventricular systolic diameter; LVEF, left ventricular ejection fraction; LAD, left atrial diameter; ACE-I, angiotensin-converting enzyme inhibitor; ARB, angiotensin-receptor blocker; SGLT, sodium-glucose linked transporter; and EAC, exhaled acetone concentration

Many kinds of blood biomarker were known in heart failure. Representative biomarker is brain natriuretic peptide. Levels of brain natriuretic peptide change during clinical course of heart failure and brain natriuretic peptide-guided treatment have benefit to reduce mortality in heart failure. ${ }^{12)}$ Unlike the blood biomarker like brain natriuretic peptide, change of breath biomarker in the clinical course of heart failure had not been clarified. Previous studies showed single exhaled acetone measurement could be diagnostic tool and could evaluate heart failure severity. ${ }^{3-6)}$ In contrast, in this preliminary study, we found out decreased exhaled acetone concentration in the improved clinical course of heart failure. Decreased exhaled acetone concentration might suggest improvement of heart failure severity and serial exhaled acetone measurements could monitor effects of treatment for heart failure. Serial exhaled acetone measurement might be a noninvasive ex- amination in the clinical course of heart failure, correlating its severity.

Acetone is a volatile component of ketone body. Exhaled acetone concentration is correlated with blood ketone bodies. Ketone bodies are significant fuel sources for the myocardium in heart failure because insulin is not required for their utilization. ${ }^{13)}$ Elevation of blood ketone bodies might be a compensated response by elevated insulin resistance in ADHF.

This study has several limitations. First, the number of subjects was relatively small, and larger prospective controlled studies are necessary to test the change of exhaled acetone concentration in patients with ADHF. Second, breath acetone needs gaschromatography to examine. Gaschromatography needs an expert to use it. To generalize breath acetone analysis in heart failure, simple measurement of breath acetone should be developed. Breath 


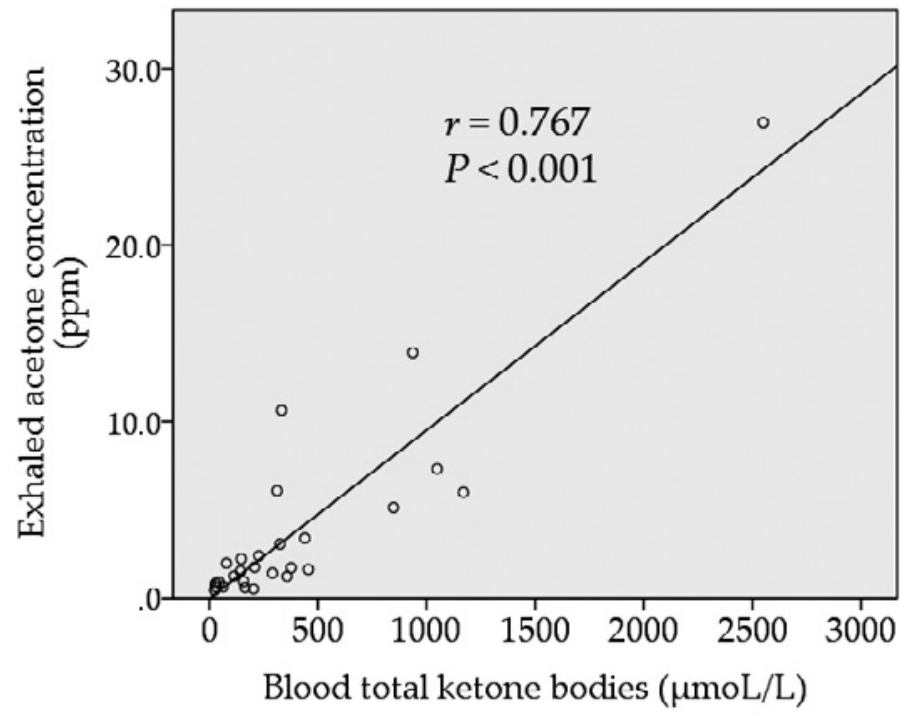

Figure 1. Correlation between blood ketone bodies and exhaled acetone concentration. Exhaled acetone concentration at the first time was correlated with blood ketone bodies in all study subjects.

Table II. Change of Exhaled Acetone Concentration

\begin{tabular}{|c|c|c|c|c|c|}
\hline & Acute phase & Stable phase & Stable phase-1 & Stable phase-2 & $P$ value \\
\hline \multicolumn{6}{|l|}{ ADHF } \\
\hline NYHA, I/II/III/IV & $0 / 0 / 14 / 5$ & $1 / 17 / 1 / 0$ & & & $<0.001$ \\
\hline BNP, pg/mL (median, IQR) & $541(292-977)$ & $431(165-679)$ & & & $<0.001$ \\
\hline EAC, ppm (median, IQR) & $2.40(1.62-6.08)$ & $0.92(0.73-1.22)$ & & & $<0.001$ \\
\hline \multicolumn{6}{|l|}{ Control } \\
\hline NYHA, I/II/III/IV & & & $3 / 4 / 1 / 0$ & $3 / 4 / 1 / 0$ & 1.000 \\
\hline BNP, pg/mL (median, IQR) & & & $420(126-640)$ & $256(34-567)$ & 0.068 \\
\hline EAC, ppm (median, IQR) & & & $0.73(0.60-0.92)$ & $0.49(0.41-0.97)$ & 0.123 \\
\hline
\end{tabular}

Values are $n$ or median (IQR). ADHF indicates acute decompensated heart failure; NYHA, New York Heart Association; $\mathrm{BNP}$, brain natriuretic peptide; IQR, interquartile range; and EAC, exhaled acetone concentration.

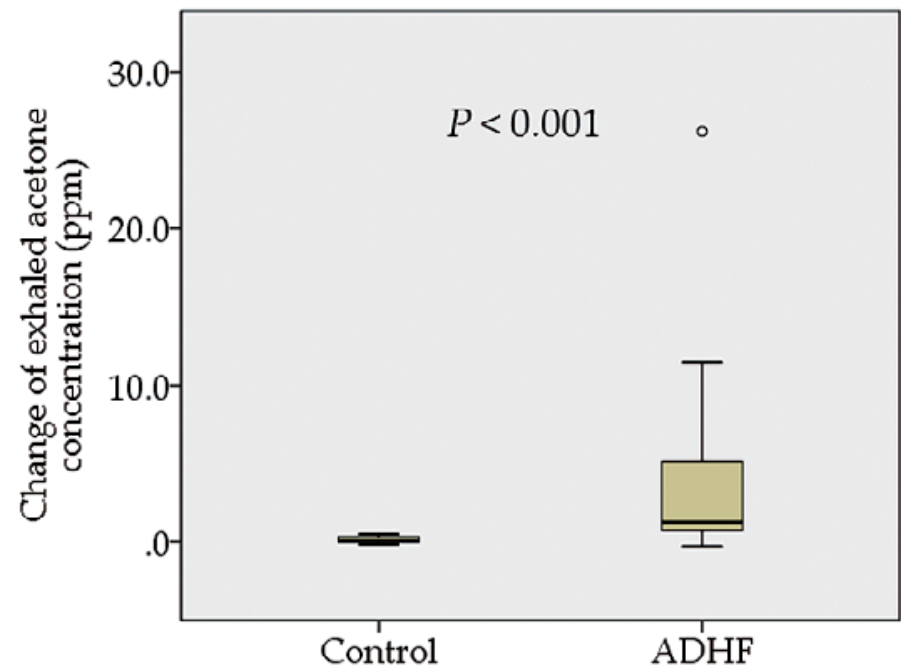

Figure 2. Comparison of the change in exhaled acetone concentration. The amount of change in exhaled acetone concentration from the first to second measurements was significantly greater in the acute decompensated heart failure (ADHF) group, compared with the control group. 
acetone concentration needs further trials to establish a position as a biomarker for heart failure.

In conclusion, exhaled acetone concentration in patients with ADHF was drastically decreased by treatment. Serial exhaled acetone measurement would be a novel noninvasive examination for the treatment of ADHF.

\section{Disclosures}

Availability of data and materials: Data are available at the Fukushima Medical University Hospital for researchers who are approved for access.

Conflicts of interest: None.

\section{References}

1. Middleton ET, Morice AH. Breath carbon monoxide as an indication of smoking habit. Chest 2000; 117: 758-63.

2. Logan RP. Urea breath tests in the management of Helicobacter pylori infection. Gut 1998; 43 Suppl 1: S47-50.

3. Marcondes-Braga FG, Gutz IGR, Batista GL, et al. Exhaled acetone as a new biomaker of heart failure severity. Chest 2012; 142: 457-66.

4. Kupari M, Lommi J, Ventilä M, Karjalainen U. Breath acetone in congestive heart failure. Am J Cardiol 1995; 76: 1076-8.

5. Samara MA, Tang WH, Cikach F Jr, et al. Single exhaled breath metabolomic analysis identifies unique breathprint in patients with acute decompensated heart failure. J Am Coll Cardiol 2013; 61: 1463-4.

6. Yokokawa T, Sugano Y, Shimouchi A, et al. Exhaled acetone concentration is related to hemodynamic severity in patients with non-ischemic chronic heart failure. Circ J 2016; 80: 117886.

7. Marcondes-Braga FG, Batista GL, Gutz IG, et al. Impact of exhaled breath acetone in the prognosis of patients with heart failure with reduced ejection fraction (HFrEF). One year of clinical follow-up. PLOS ONE 2016; 11: e0168790.

8. Yokokawa T, Sugano Y, Shimouchi A, et al. A case of acute decompensated heart failure evaluated by series of exhaled acetone concentrations as noninvasive biomarker of heart failure severity. Int J Cardiol 2016; 204: 112-3.

9. Yokokawa T, Ichijo Y, Houtsuki Y, et al. Change of exhaled acetone concentration in a diabetic patient with acute decompensated heart failure. Int Heart J 2017; 58: 828-30.

10. McKee PA, Castelli WP, McNamara PM, Kannel WB. The natural history of congestive heart failure: the Framingham study. N Engl J Med 1971; 285: 1441-6.

11. Lang RM, Badano LP, Mor-Avi V, et al. Recommendations for cardiac chamber quantification by echocardiography in adults: an update from the American Society of Echocardiography and the European Association of Cardiovascular Imaging. J Am Soc Echocardiogr 2015; 28: 1-39.e14.

12. Porapakkham P, Porapakkham P, Zimmet H, Billah B, Krum H. B-type natriuretic peptide-guided heart failure therapy: A metaanalysis. Arch Intern Med 2010; 170: 507-14.

13. Aubert G, Martin OJ, Horton JL, et al. The failing heart relies on ketone bodies as a fuel. Circulation 2016; 133: 698-705. 